AIAA 96-0825

Aerodynamic Characteristics of an Aerospace Vehicle During a Subsonic Pitch-Over Maneuver

William L. Kleb

NASA Langley Research Center

Hampton, Virginia 


\title{
Aerodynamic Characteristics of an Aerospace Vehicle During a Subsonic Pitch-Over Maneuver
}

\author{
William L. Kleb* \\ NASA Langley Research Center, Hampton, Virginia, 23681-0001
}

\begin{abstract}
Time-dependent CFD has been used to predict aerospace vehicle aerodynamics during a subsonic rotation maneuver. The inviscid $3 \mathrm{D} 3 \mathrm{U}$ code is employed to solve the 3-D unsteady flowfield using an unstructured grid of tetrahedra. As this application represents a challenge to time-dependent CFD, observations concerning spatial and temporal resolution are included. It is shown that even for a benign rotation rate, unsteady aerodynamic effects are significant during the maneuver. Possibly more significant, however, the rotation maneuver creates flow asymmetries leading to yawing moment, rolling moment, and side force which are not present in the quasi-steady case. A series of steady solutions at discrete points in the maneuver are also computed for comparison with wind tunnel measurements and as a means of quantifying unsteady effects.
\end{abstract}

\section{Introduction}

NASA's Access to Space Study ${ }^{1}$ recommended the development of a fully Reusable Launch Vehicle (RLV) ${ }^{2}$ to replace the aging Space Shuttle. A method of reaching this goal is to develop a vehicle which does not rely on expendable boosters to reach orbit, a so-called SingleStage-to-Orbit (SSTO) vehicle. ${ }^{3}$ One of the SSTO configurations being investigated is the Vertical Take-off and Vertical Landing (VTVL) configuration. ${ }^{3}$ In one concept, the VTVL vehicle, upon completion of it's mission in low Earth orbit, reenters nose first, decelerates to subsonic speeds, and then performs a rotation maneuver ${ }^{4-6}$ to land vertically.

Figure 1 presents a schematic of the last portion of a typical VTVL entry. The pitch-over maneuver, which occurs near Mach 0.2 , is characterized by high angle of attack, unsteady, vortical flow. Accurately predicting vehicle performance during this aerodynamic pitch-over maneuver is quite challenging. While ground-based facilities can predict the vehicle's aerodynamics at discrete points during the maneuver, accounting for the unsteady effects in a wind tunnel is difficult. ${ }^{7}$ Time-dependent Computational Fluid Dynamics (CFD) offers another means of analyzing the pitch-over maneuver. The ma-

*Research Engineer, Aerothermodynamics Branch, Gas Dynamics Division, Research Technology Group.

Copyright (C) 1996 by the American Institute of Aeronautics and Astronautics, Inc. No copyright is asserted in the United States under Title 17, U. S. Code. The U. S. Government has a royalty-free license to exercise all rights under the copyright claimed herein for Governmental purposes. All other rights are reserved by the copyright owner.

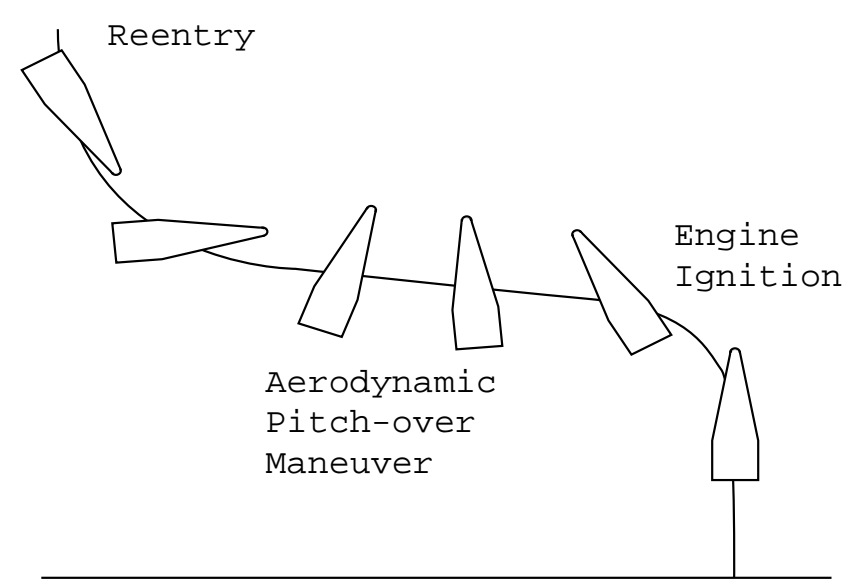

Figure 1: Transition to landing for a VTVL vehicle.

jority of work in unsteady CFD has, however, been restricted to small amplitude, harmonic variations in angle of attack in support of aeroelastic flutter predictions. ${ }^{8}$

The objective of the present work is to apply unsteady CFD methods to the problem of predicting VTVL vehicle aerodynamics during an un-powered pitch-over maneuver. The inviscid 3D3U code of Batina ${ }^{9}$ is employed to solve the $3-\mathrm{D}$ unsteady flowfield with unstructured grids. As this application represents a new challenge to unsteady CFD, observations concerning grid resolution, temporal resolution and the suitability of assuming flowfield symmetry are discussed. A series of steady solutions at discrete points in the maneuver are also computed for comparison with wind tunnel measurements and as a means of quantifying unsteady effects. 


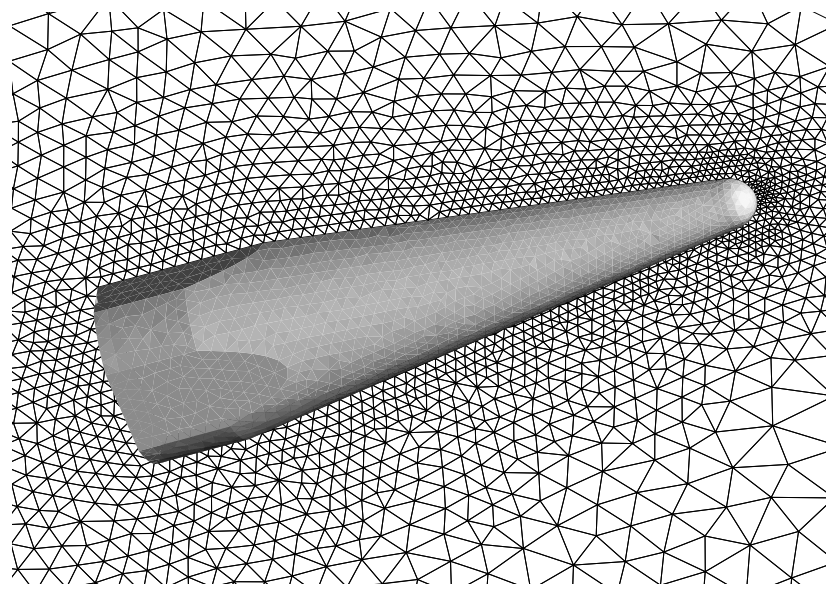

Figure 2: VTVL vehicle shown with a coarse symmetry plane mesh.

\section{Geometry}

Figure 2 presents the geometry examined. The forebody is an 8 degree half-angle sphere cone with nose radius equal to 0.3 of the base radius. The aft body, beginning at the 85 percent fuselage station, is a cylinder with a partially squared-off cross-section producing flat "slices" extending from the base of the vehicle to approximately the 60 percent fuselage station. The geometry has been non-dimensionalized so that half the width of the base is one unit and the radius of the cylindrical portion is 1.12 . The vehicle is 7.16 units long, giving a fineness ratio of 6.4 using a base diameter of 2.24. A more thorough description of the vehicle is given by Woods. ${ }^{10}$

\section{Computational Mesh}

The underlying surface definition database was generated from structured surface patches obtained using GRIDGEN,${ }^{11}$ GridTool, ${ }^{12}$ and simple analytical methods. The unstructured surface and flowfield grids were then generated using FELISA $^{13}$ and TETMESH. ${ }^{14}$

Two different options for the computational domain were employed: one modeling the complete vehicle and another modeling only half of the vehicle, assuming symmetry across the pitch-plane (see Figure 2). Table 1 details the symmetric-vehicle grids, showing number of points, etc., while Table 2 provides similar information for the full-vehicle grids. All of the results shown in this paper are the result of using the coarsest meshes: Mesh $1 \mathrm{~F}$ and Mesh 1S. This is for the sake of consistency and completeness since the matrix of runs on the finer meshes is not as complete as that on the coarser meshes due to resource limitations. Some of the finer mesh solutions are, however, incorporated in the grid resolution results.
Table 1: Symmetric-vehicle grids.

\begin{tabular}{lrr}
\hline \hline & Mesh 1S & Mesh 2S \\
\hline Nodes & 6,634 & 27,912 \\
Tetrahedra & 32,374 & 157,599 \\
Boundary nodes & 2,596 & 5,183 \\
Boundary triangles & 5,188 & 10,362 \\
Average vehicle spacing & 0.1 & 0.1 \\
Average farfield spacing & 3.5 & 3.5 \\
Radius of farfield & 3.5 & 3.5 \\
\hline \hline
\end{tabular}

Table 2: Full-vehicle grids.

\begin{tabular}{lrr}
\hline \hline & Mesh 1F & Mesh 2F \\
\hline Nodes & 11,222 & 51,179 \\
Tetrahedra & 60,597 & 308,108 \\
Boundary nodes & 2,781 & 3,789 \\
Boundary triangles & 5,554 & 7,570 \\
Average vehicle spacing & 0.1 & 0.1 \\
Average farfield spacing & 3.5 & 3.5 \\
Radius of farfield & 3.5 & 3.5 \\
\hline \hline
\end{tabular}

\section{Far-Field Boundary}

Even with non-reflecting boundary conditions, farfield boundary placement can affect the solution for bodies producing lift. For the results presented, the farfield boundary condition is applied on a sphere which is $3-1 / 2$ body lengths from the vehicle's center. As a spot-check of the effect of the far-field boundary placement, two other grids were generated with the far-field location at 5 and 7 body lengths from the vehicle's center. At moderate-to-high angles of attack, the change in far-field placement produced no appreciable affect on the aerodynamic coefficients; thus, the 3.5 body length meshes were used to minimize the resources necessary for the solution.

\section{Symmetry Assumptions}

Flowfields about symmetric configurations at high angles of attack, even with zero side-slip, often involve asymmetric, vortex-dominated, flowfields. ${ }^{15-18}$ As reviewed by Newsome et al, ${ }^{19}$ inviscid simulations can offer some indication of this viscous-dominated phenomenon through the numerical viscosity required by the scheme. Hence, solutions are compared for both steady and unsteady conditions modeling the complete vehicle and those which impose flowfield symmetry across the pitchplane. 


\section{Numerical Method}

The 3D3U code of Batina ${ }^{9}$ was used exclusively in this study. The 3D3U code was originally developed to study harmonically pitching wings and wing-bodies in transonic flow. The code can incorporate aeroelastic effects through assumed mode shapes, coupled with a deforming mesh via the linear spring analogy.

Inviscid fluxes can be computed with any of the following flux functions: Roe's flux-difference splitting, ${ }^{20}$ Lou and Steffen's AUSM scheme, ${ }^{21}$ van Leer's fluxvector splitting, ${ }^{22}$ or Einfeldt's HLLE scheme. ${ }^{23}$ Secondorder flux reconstruction is achieved via van Leer's MUSCL (a.k.a Kappa) scheme ${ }^{24}$ using node averaged variables according to Batina $^{9}$ or Frink ${ }^{25}$ with Mulder and van Leer's continuously-differentiable, flux limiting. ${ }^{26}$ This provides global, second-order, spatial accuracy for all flux functions except the HLLE flux function which cannot use higher order reconstruction of this form.

First-order temporal accuracy in a global sense is accomplished through forward Euler time stepping with any of three implicit time integration schemes: ${ }^{9}$ GaussSeidel, Jacobi, and Point-Jacobi; or the explicit, MStage, Runge-Kutta time stepping scheme of Turkel, Schmidt, and Jameson. ${ }^{27}$

For this study, the code was applied to a transient, large-amplitude motion at a fairly low Mach numberrepresenting a significant departure from cases which the code was originally intended. Due to this new, demanding application, a grid study and a time-step study will be presented.

The following defaults were used for the computed results in this study, unless otherwise noted: Roe's fluxdifference splitting, an eigenvalue limiter threshold value of 0.3 , second-order flux reconstruction using a $\kappa$ of 0.5 , and Gauss-Seidel implicit time integration with a CFL number of one million.

\section{Coefficient Definitions}

Describing the aerodynamics of the VTVL vehicle during the pitch-over maneuver requires specification of the force and moment coefficients as a function of angle of attack. The aerodynamic coefficients: normal force, $C_{N}$, axial force, $C_{A}$, and pitching moment, $C_{m}$, are defined relative to the vehicle's stability axes as shown in Figure 3(a). The normal force coefficient, $C_{N}$, is positive in the z-direction while the axial force coefficient, $C_{A}$, is defined positive in the $\mathrm{x}$-direction. Using the right hand rule, crossing the $\mathrm{z}$-axis into the $\mathrm{x}$-axis, gives the positive orientation for the pitching moment coefficient, $C_{m}$. The remaining aerodynamic coefficients: side force, $C_{Y}$, and yawing moment, $C_{n}$ are defined as shown in Figure 3(b).

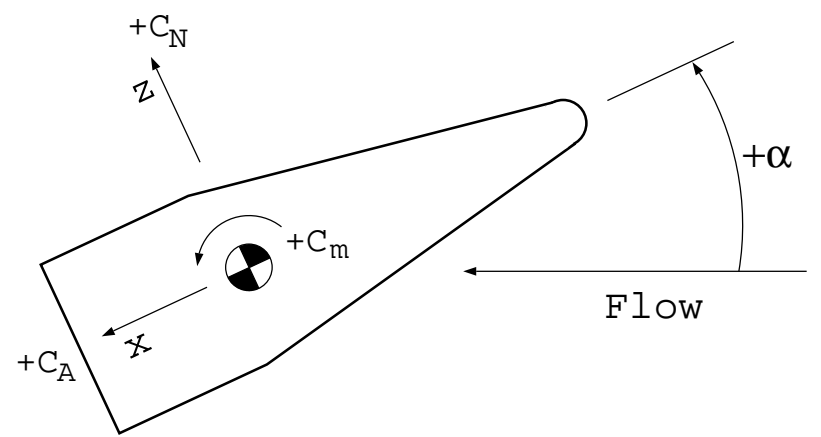

(a) Normal and axial forces, pitching moment.

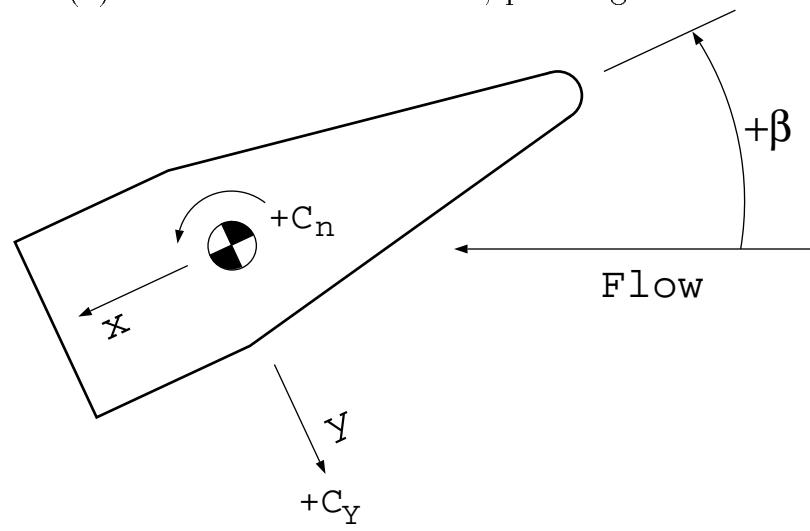

(b) Side force and yawing moment.

Figure 3: Orientation of force and moment coefficients.

A positive rolling moment, $C_{l}$, is given by the right hand rule about the $\mathrm{x}$-axis.

For this vehicle the stability axes, located at the center of gravity, was given as $(4.3885,0,0)$. The reference length for the moment coefficients is the base radius, 1.12 ; and the reference area for all the non-dimensional coefficients, using the base radius, is 3.94 .

\section{Pitch-Rate Schedule}

The pitch schedule chosen for this study is shown in Figure 4. The maneuver is the first half of a sine function. The vehicle is assumed to be in steady flight at 17.5 degrees angle of attack, Mach 0.2. At time zero, the vehicle begins the pitch-over maneuver, reaching a maximum pitch rate exactly half way through the maneuver and finishing at a 180 degree angle of attack. For this study, the time to complete the maneuver, $T_{f}$, was chosen as 90 seconds, giving a maximum rotation rate of 3.1 degrees per second at 45 seconds through the maneuver. For simplicity, it is assumed that the freestream Mach number (0.2) remains constant throughout the maneuver. 


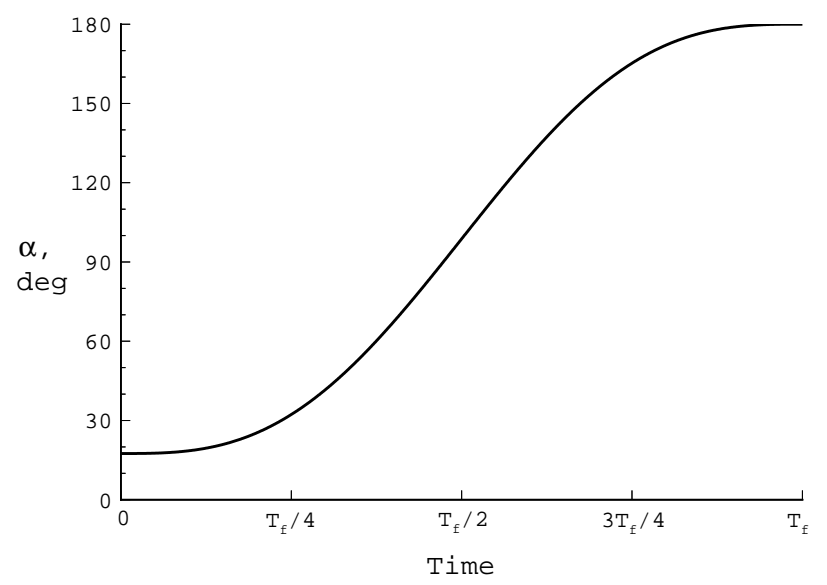

(a) Angle-of-attack.

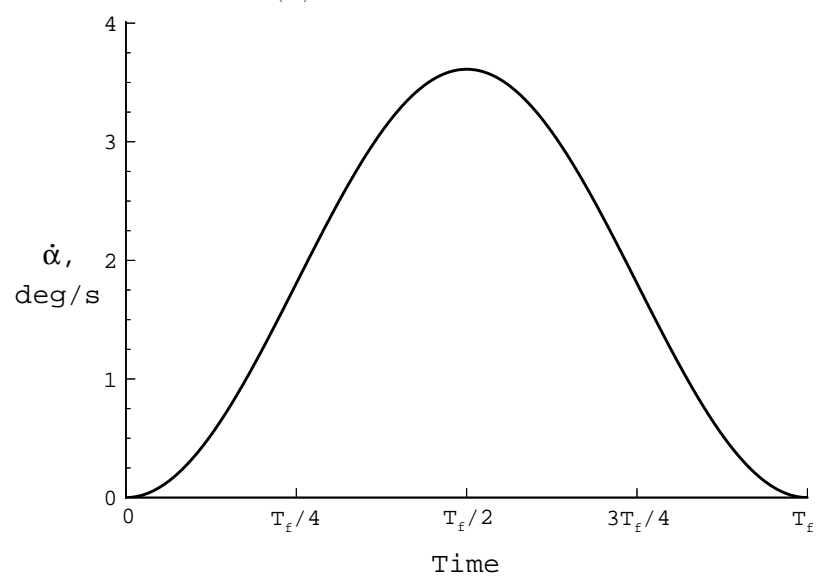

(b) Pitch rate.

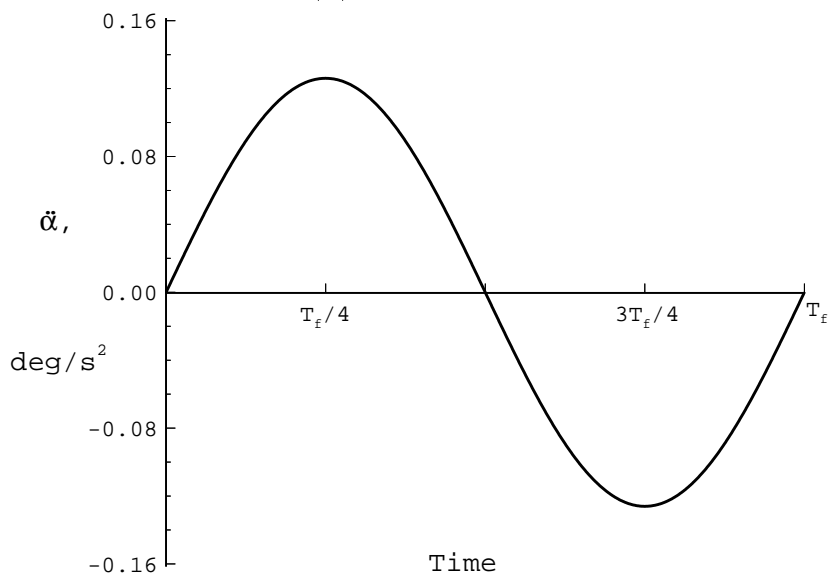

(c) Pitch acceleration.

Figure 4: Definition of pitch-over maneuver.

\section{Resolution Studies}

Since this is the first time the 3D3U code has been run to predict a transient motion, both a grid convergence and time step convergence study were performed.

\section{Spatial}

Figure 5 represents steady results for an angle of attack range from 0 to 90 degrees using a coarse and a fine grid. As alluded to earlier, the effects of grid resolution on the separation lines is significant. This is due to the fact that this is an "inviscid" simulation and the

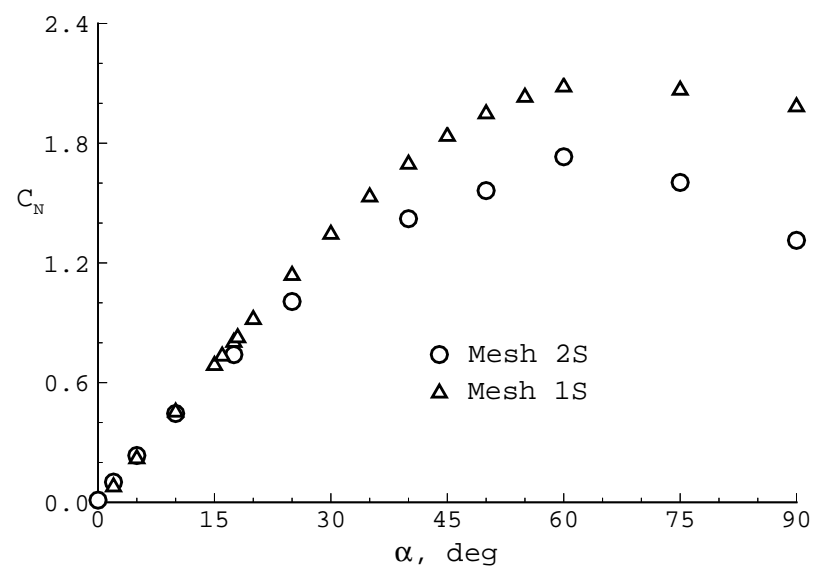

(a) Normal force coefficient.

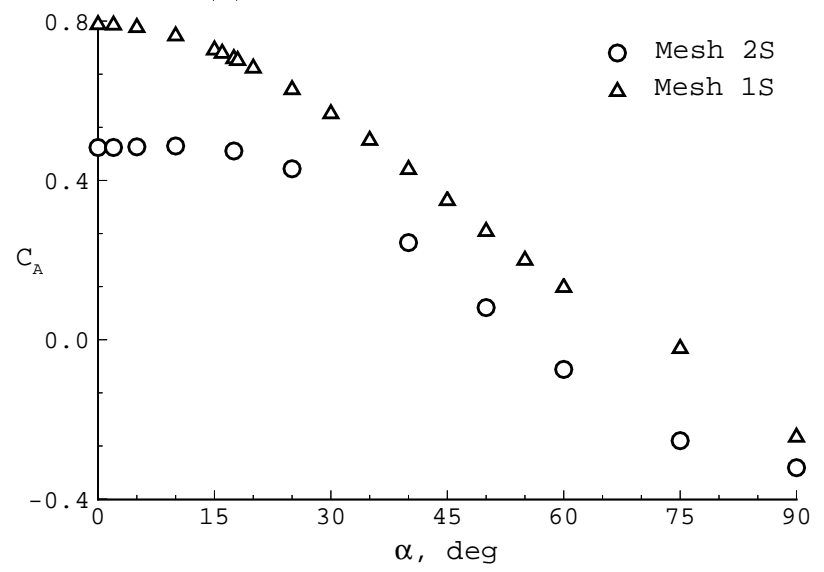

(b) Axial force coefficient.

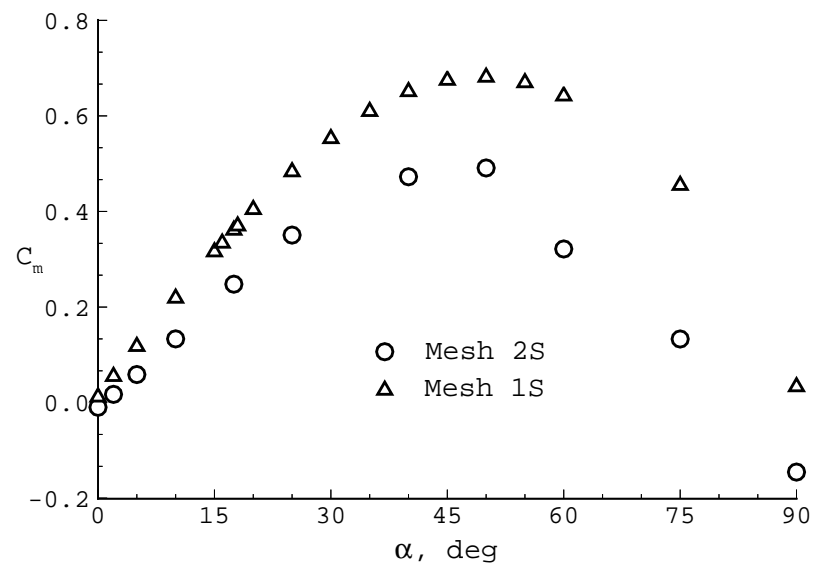

(c) Pitching moment coefficient.

Figure 5: Grid convergence study. 
only viscosity present is due to the numeric discretization; and, as the grid is refined, the amount of numerical viscosity decreases, yielding a more nearly inviscid solution. Since the flow takes longer to separate on the refined grid due to less numerical dissipation, the force coefficients are lowered due to the smaller area of low pressure, separated flow on the leeward portions of the vehicle.

As the figure clearly shows, the solution is not gridconverged by any measure. The question of grid convergence of an inviscid solution for a flow problem involving separated flow does not have a true answer since the governing equations are not correct. Without modeling the Navier-Stokes equations, the solutions obtained can, and should only be used qualitatively.

\section{Temporal}

Figure 6 shows effect of successively refining the time step. The three time steps chosen were $1 / 60$ th, $1 / 120$ th, and $1 / 240$ th of a second. (The $1 / 120$ th data is the unmarked line bounded by the $1 / 60$ th and $1 / 240$ th lines-only disguishable around 45 degrees angle of attack.) Since the time stepping scheme is formally firstorder accurate and the code was run in an implicit timestepping mode, a significant effect was expected for variation of the time step; but, as Figure 6 shows, the only effect occurred in the 30 to 60 degree angle-of-attack range of the maneuver. This implies that except for that region, the initial time step chosen was small enough to resolve the flow in a global sense. The time-dependent results presented were computed using the $1 / 240$ th second time step and are considered to be temporarily converged.

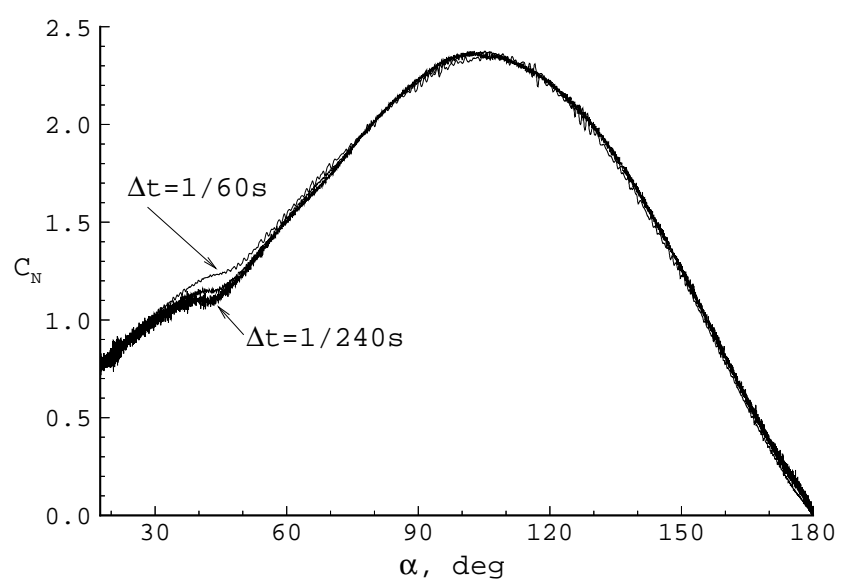

Figure 6: Time step convergence study: normal force coefficient for various time steps, Mesh $1 \mathrm{~F}$.

\section{Results}

\section{Steady Flow}

As a means to examine the unsteady effects of the pitch-over maneuver itself, steady flow at selected angles of attack were computed. Figure 7 shows a comparison of two aerodynamic coefficients with the experimental data of Woods ${ }^{10}$ for angles of attack from 0 to 60 degrees. The computed results agree well at small angles of attack, and diverge from the experimental results as the angle of attack increases due to the different position of the leeside separation line-a viscous phenomenon. As discussed earlier, since the computed results are modeling inviscid flow the only mechanism for flow separation is the numerical dissipation in the scheme. Thus, the exact location of the computed separation line is highly grid and scheme dependent. Since the leeside separation line runs almost the entire length of the vehicle, a slight deviation can make a large difference in the integrated coefficients.

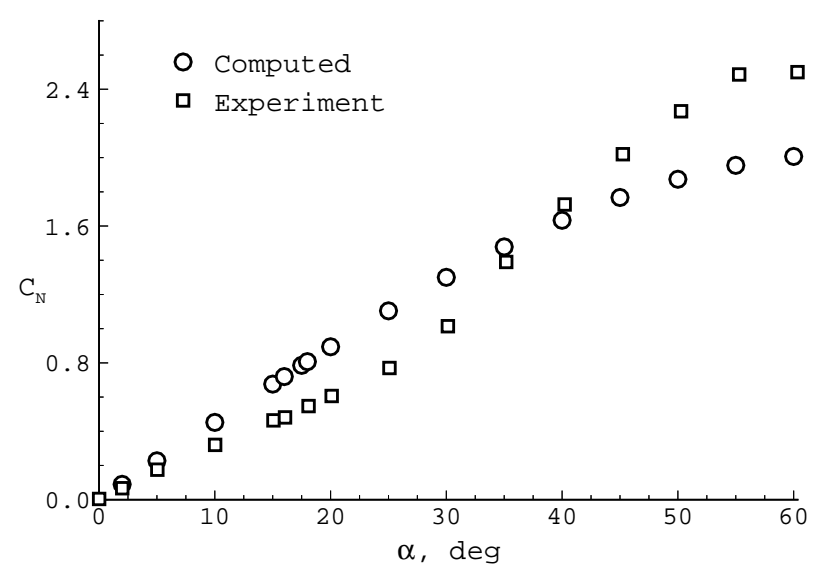

(a) Normal force coefficient.

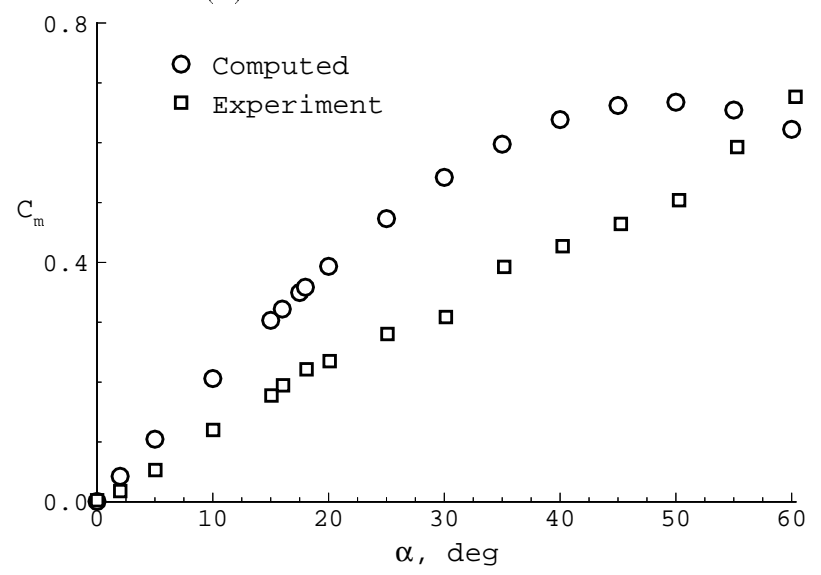

(b) Pitching moment coefficient.

Figure 7: Comparison of steady results with experiment of Woods. ${ }^{10}$ 


\section{Unsteady Flow}

Figure 8 compares aerodynamic coefficients from both steady and unsteady calculations. The solid line represents the unsteady results and the symbols are

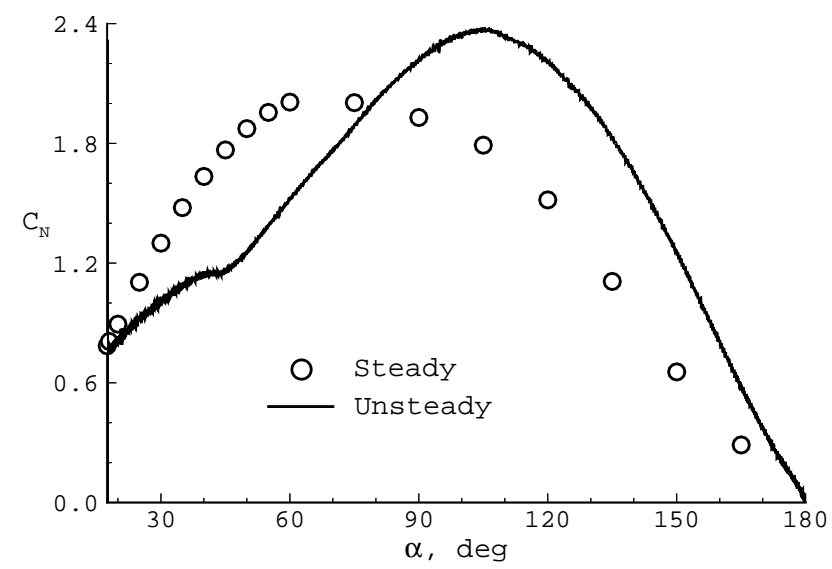

(a) Normal force coefficient.

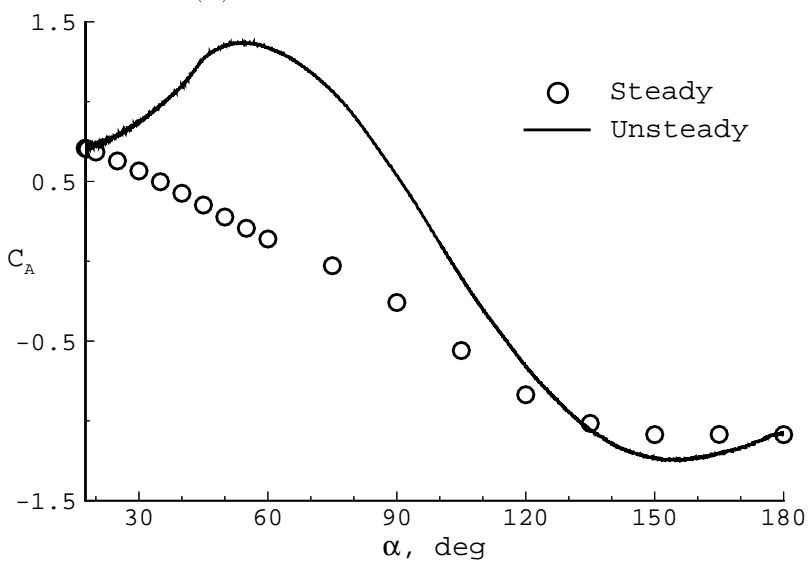

(b) Axial force coefficient.

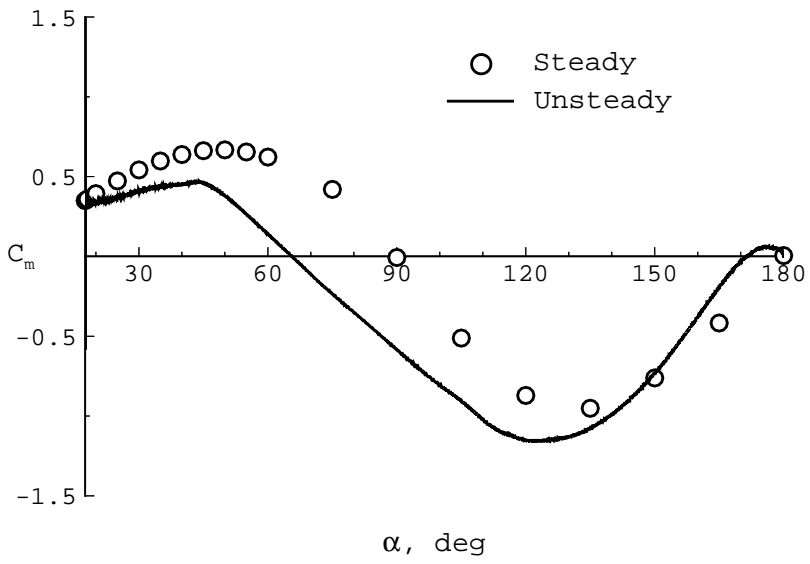

(c) Pitching moment coefficient.

Figure 8: Comparison of steady and unsteady aerodynamic coefficients. the steady flow results. (Recall Figure 4 for the pitch rates and accelerations corresponding to each angle of attack.) Readily discernible is that the steady and unsteady aerodynamic coefficients are significantly different. The pitching moment coefficient appears to have the least quantifiable deviation, whereas the unsteady axial force coefficient has a completely different qualitative pattern than that for quasi-steady flow. The normal force coefficient appears to have the time lag behavior expected in moderately unsteady flow: showing the same general qualitative trend throughout the angle-of-attack range, but with the unsteady results lagging behind the steady results.

As a method of more closely examining the integrated aerodynamic coefficients' behavior, coefficient of pressure contours are shown in Figure 9. This figure shows contours on the vehicle and on the pitch plane for several angles of attack. The steady results are shown on the left and the instantaneous, unsteady results are shown on the right. Most notable is the side of the vehicle's forebody: the unsteady case has consistently higher pressures than the steady case at all angles of attack. A similar trend is also reflected in the symmetry plane contours: here the unsteady case has a generally higher pressure where the vehicle is rotating into the oncoming flow with a more severe expansion in the base region as compared to the steady results.

\section{Flow Asymmetry}

Figure 10 shows a comparison between a symmetrically modeled vehicle and a fully modeled vehicle for an angle-of-attack range between 20 and 60 degrees for steady flow. As borne out by the figure, within the accuracy of the computation, there appear to be no appreciable asymmetries in the steady flow. (Note the expanded scales for the side force and yawing moment coefficient.)

However, for the unsteady case, asymmetric flow is apparent in Figure 11. This figure shows differences between symmetric vehicle and full vehicle for unsteady flow. Figure 11 also shows the appearance of a side-force, yawing moment, and rolling moment previously reported by Woods ${ }^{10}$ although at much lower overall values. In addition, for this study, the most significant manifestations of the asymmetries occur in the 90 to 135 degree angle of attack range whereas the most pronounced differences in the normal force coefficient occur in the 30 to 60 degree range. (Note: Woods ${ }^{10}$ did not perform tests above 60 degrees angle of attack for this geometry and flow condition.)

\section{Concluding Remarks}

The objective of the present work was to focus an unsteady CFD method on the problem of predict- 


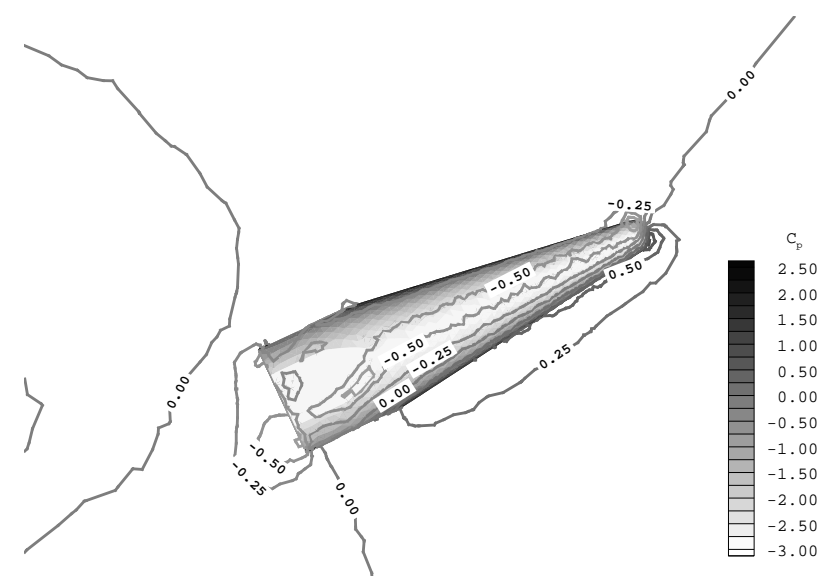

(a) Steady, $\alpha=30^{\circ}$.

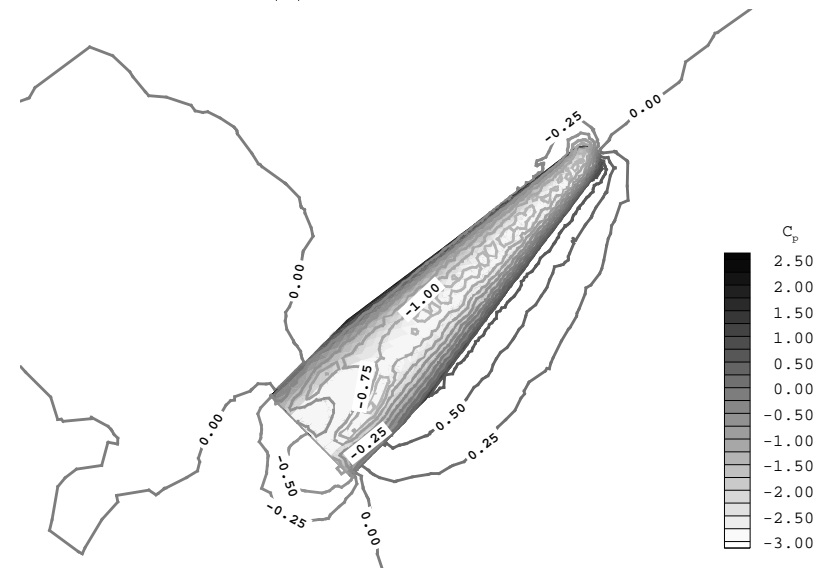

(c) Steady, $\alpha=50^{\circ}$.

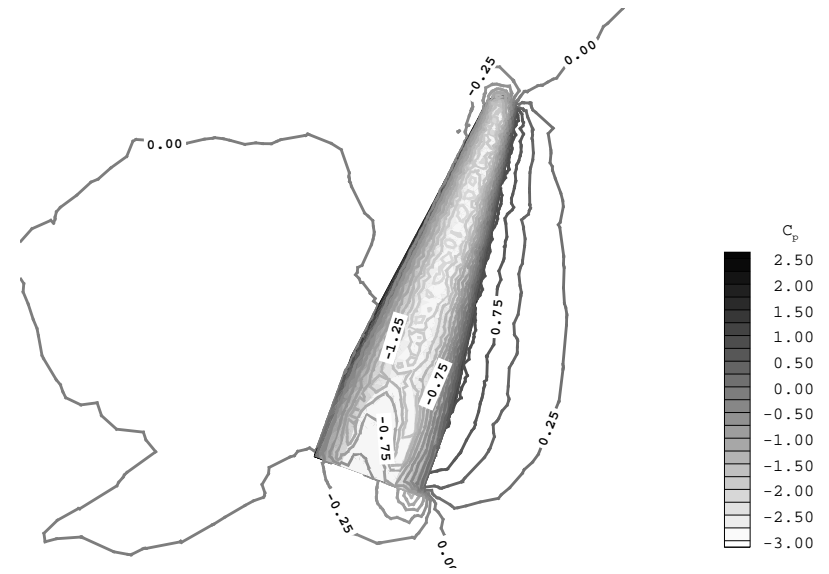

(e) Steady, $\alpha=75^{\circ}$.

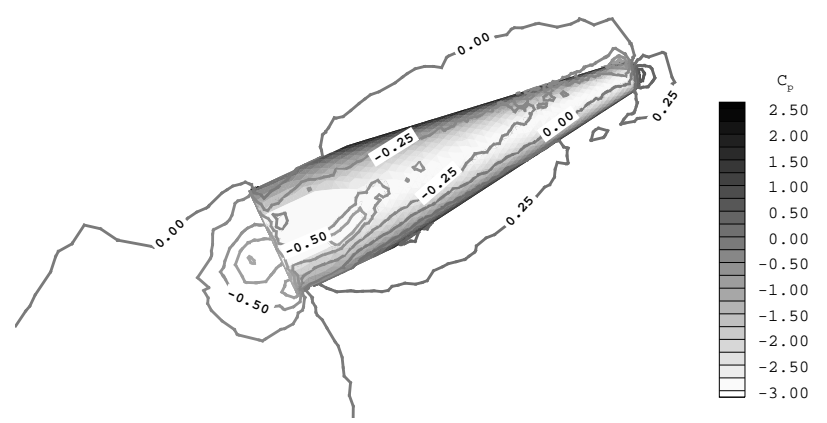

(b) Unsteady, $\alpha=30^{\circ}$.

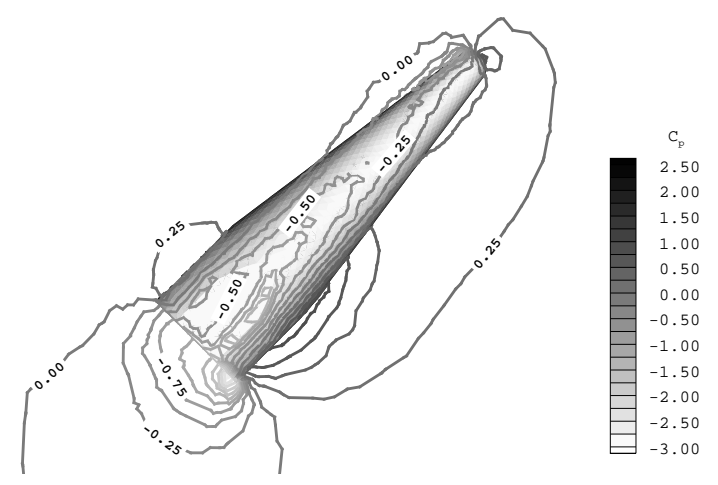

(d) Unsteady, $\alpha=50^{\circ}$.

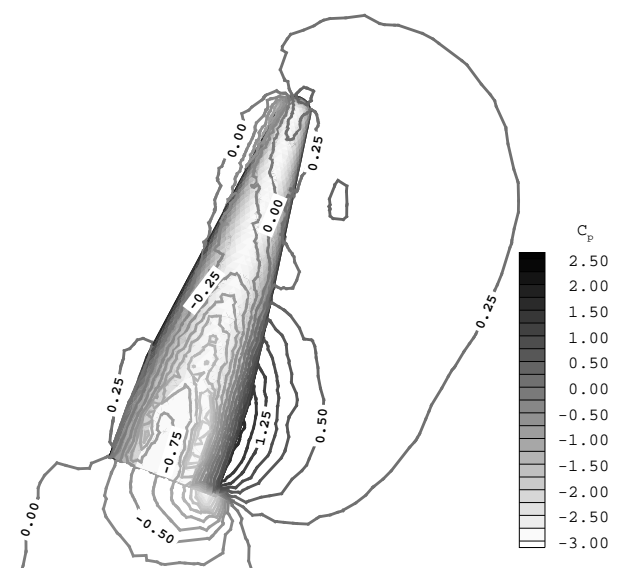

(f) Unsteady, $\alpha=75^{\circ}$.

Figure 9: Comparison of steady and unsteady coefficient of pressure distributions. 


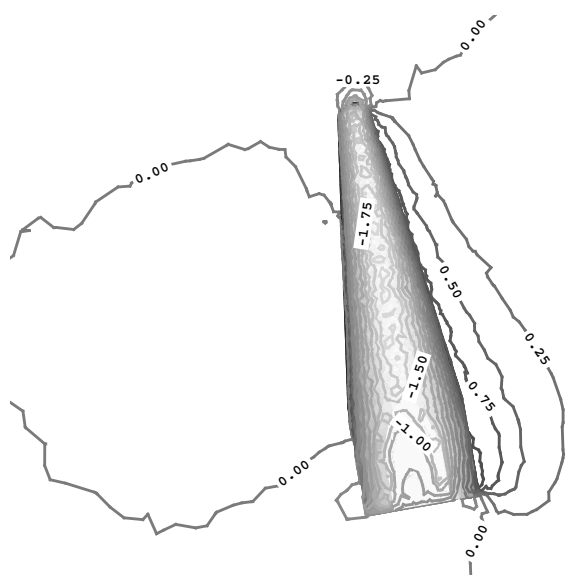

(g) Steady, $\alpha=105^{\circ}$.

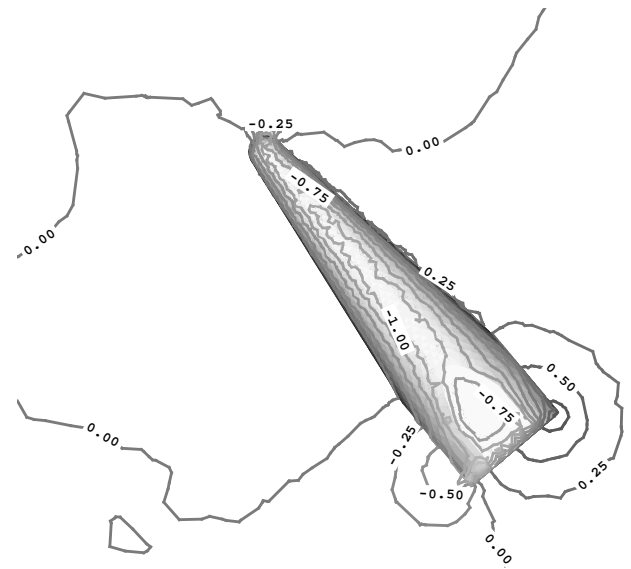

(i) Steady, $\alpha=135^{\circ}$.

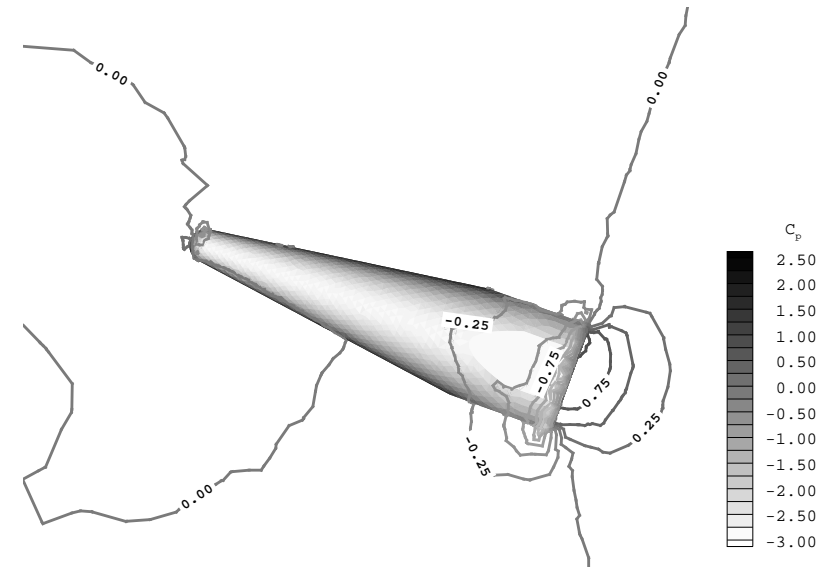

(k) Steady, $\alpha=165^{\circ}$.

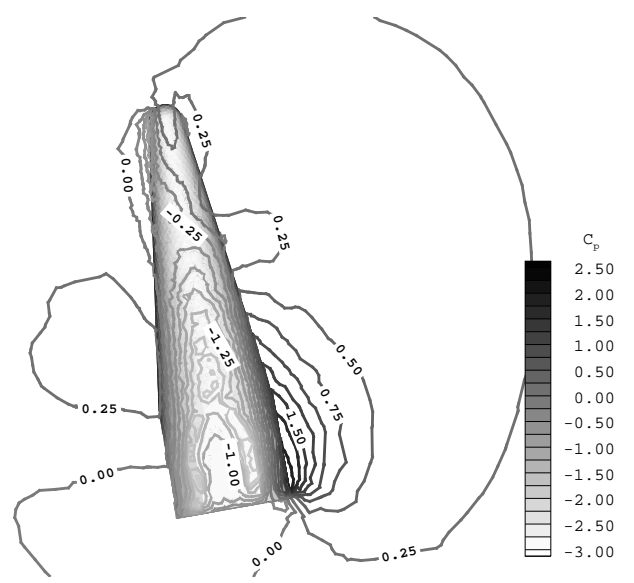

(h) Unsteady, $\alpha=105^{\circ}$.

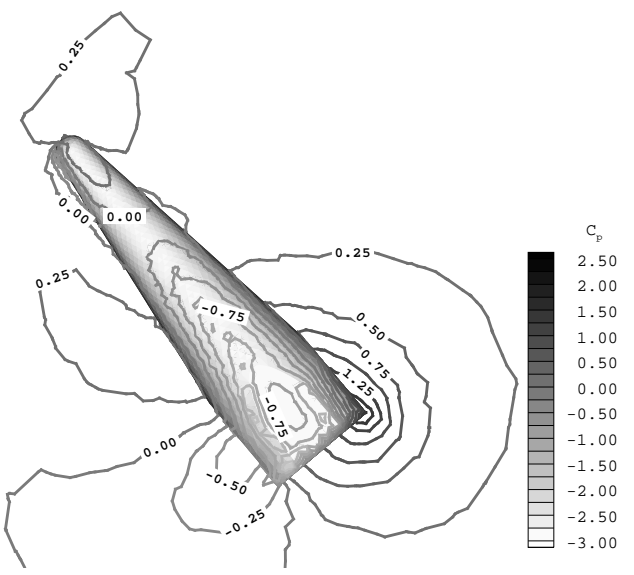

(j) Unsteady, $\alpha=135^{\circ}$.

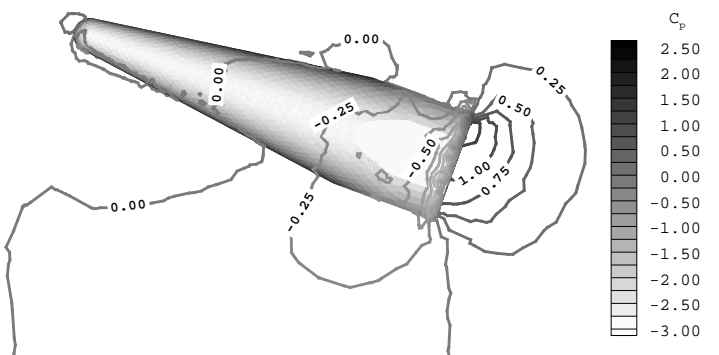

(l) Unsteady, $\alpha=165^{\circ}$.

Figure 9: Concluded. 


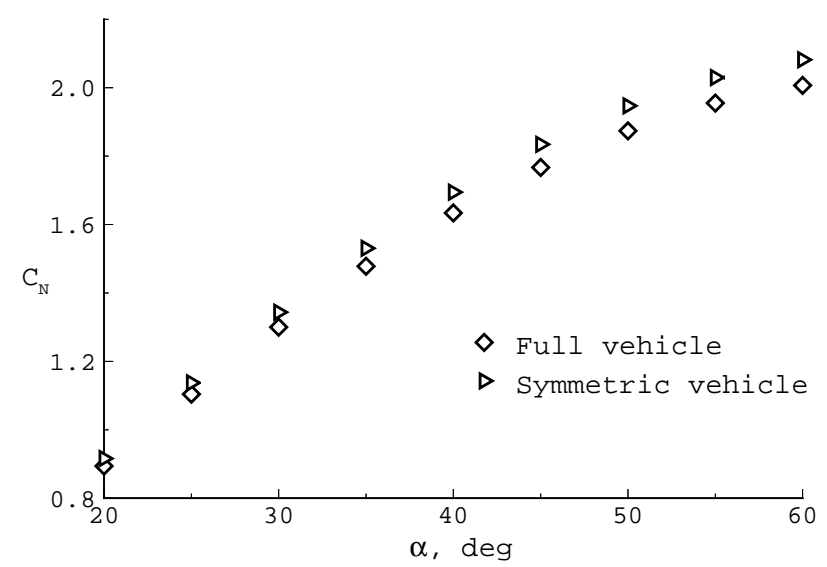

(a) Normal force coefficient.

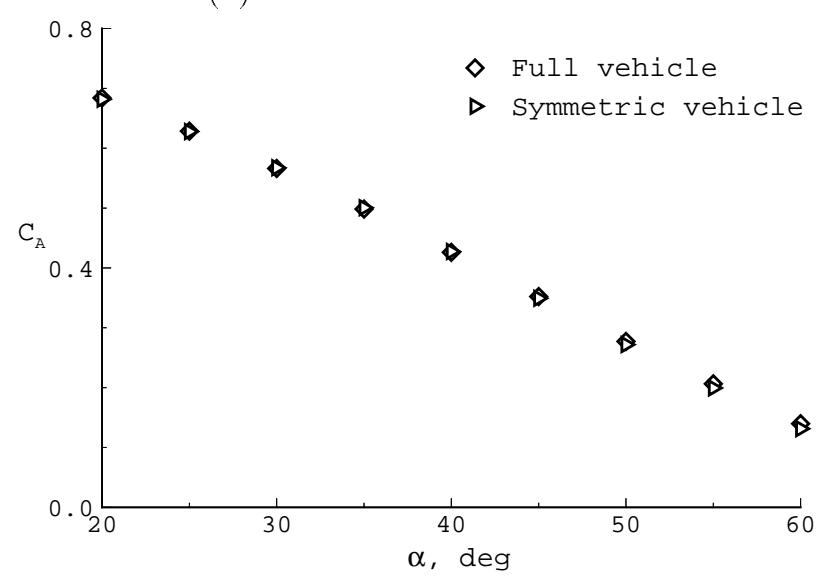

(c) Axial force coefficient.

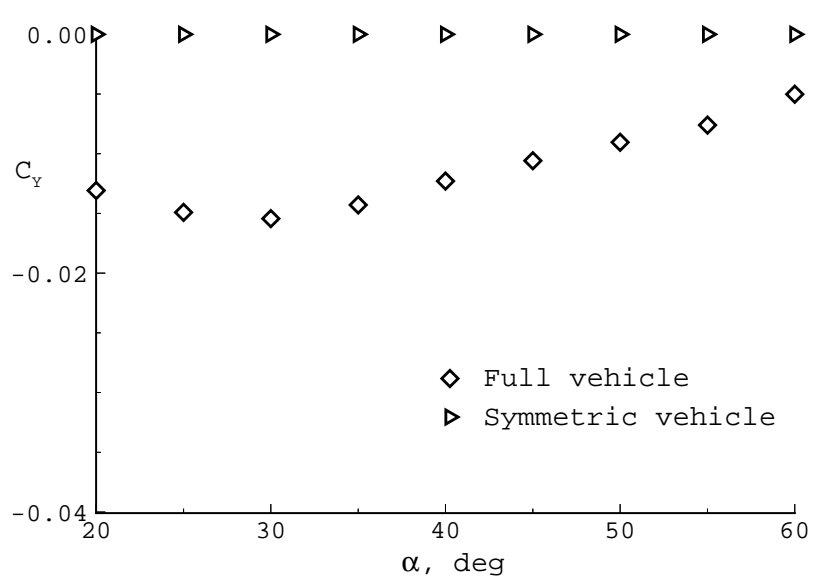

(b) Side force coefficient.

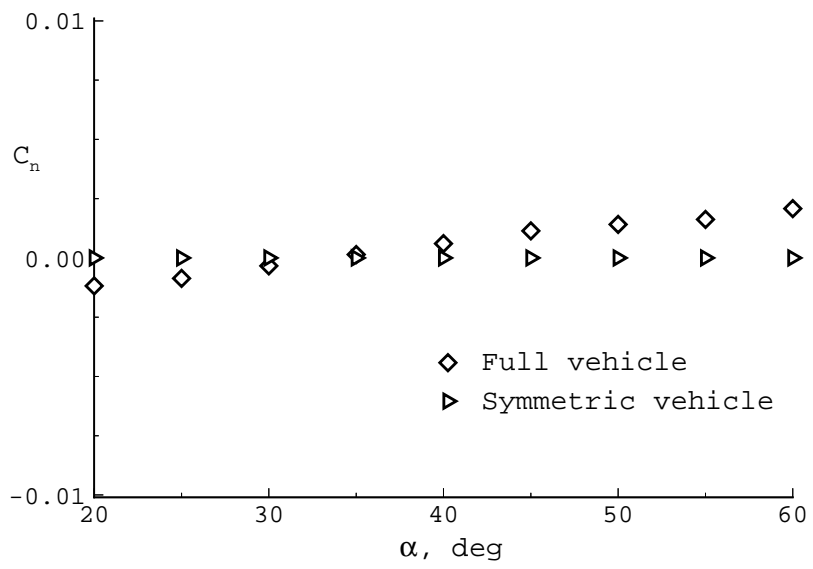

(d) Yawing moment coefficient.

Figure 10: Steady asymmetries in the flow.

ing VTVL vehicle aerodynamics during the pitch-over maneuver. This was accomplished through the use of the inviscid 3D3U code with unstructured grids. Even though the simulation was performed using an inviscid flow solver, predictions for steady-state cases agreed with experimental results at the low angles of attack. A series of steady solutions at discrete points in the maneuver were computed and it was shown that even for the unrealistically slow pitch-over rate studied, unsteady effects were large. More importantly, the rotation maneuver appears to create flow asymmetries leading to yawing moments, rolling moments, and side forces which are not significant in the quasi-steady case.

As this is an exploratory study, there is certainly room for future work. The following is just a handful of extensions which would be necessary to create an effective design tool: incorporating viscous effects, allowing movable control surfaces, coupling a six-degree-offreedom rigid body dynamics solver, adding control law, and possibly incorporating grid-adaptive capabilities.

\section{Acknowledgments}

Thanks are extended to Scott Ward and John Copper of McDonnell Douglas Space Systems Company, Huntington Beach, California, for providing the concept geometry used in this study.

Dr. Neil Cheatwood of Vigyan, Hampton, Virginia provided the surface geometry patch definition which was used as input for the unstructured grid generation; and, Dr. Ramadas Prabhu of Lockheed Engineering and Sciences Corporation, Hampton, Virginia, generated the unstructured surface and volume grids which using the FELISA package. ${ }^{13}$

Thanks are also extended to Dr. John Batina, Dr. Neal Frink, Dr. Robert Mitcheltree, and Robert Scott of NASA Langley Research Center, Hampton, Virginia, as well as to Dr. Dimitri Mavriplis, of ICASE, Hampton, Virginia, and Dr. Russ Rausch of Lockheed 


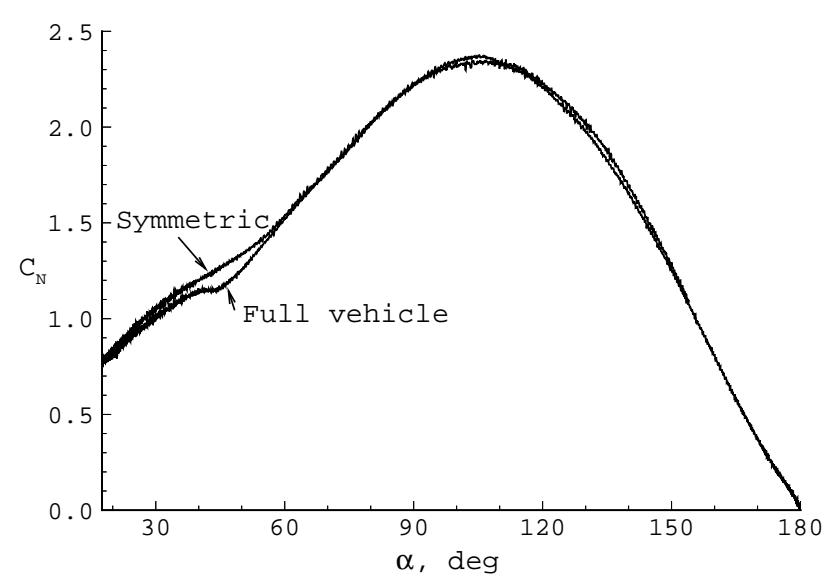

(a) Normal force coefficient.

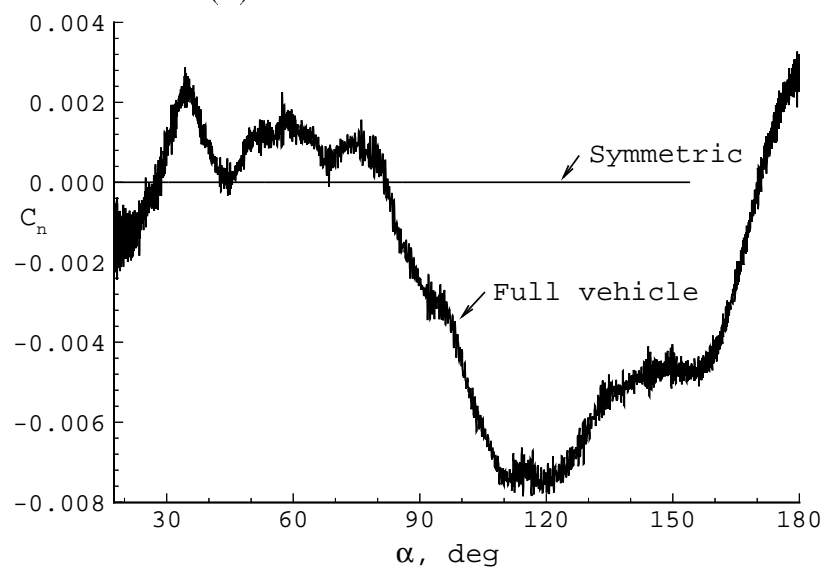

(c) Yawing moment coefficient.

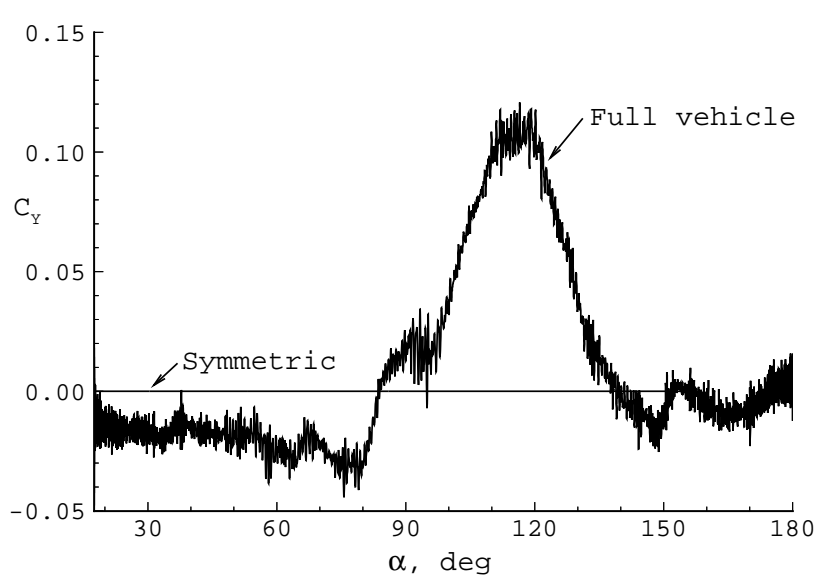

(b) Side force coefficient.

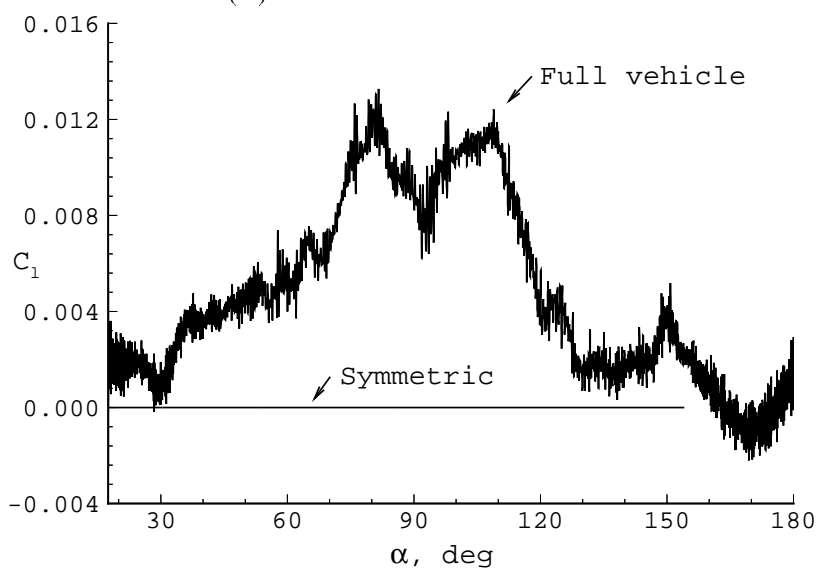

(d) Rolling moment coefficient.

Figure 11: Unsteady asymmetries in the flow.

Engineering and Sciences Corporation, Hampton, Virginia, for their helpful discussions throughout this study.

\section{References}

${ }^{1}$ Bekey, I., Powell, R., and Austin, R., "NASA Studies Access to Space," Aerospace America, May 1994, pp. $38-43$.

${ }^{2}$ Dornheim, M. A., "NASA Awards RLV Contracts," Aviation Week \& Space Technology, June 27 1994, pp. 22-23.

${ }^{3}$ Austin, R. E., and Cook, S. A., "SSTO Rockets: Streamlining Access to Space," Aerospace America, November 1994, pp. 34-38.

${ }^{4}$ Dornheim, M. A., "DC-X Demonstrates Key Maneuver," Aviation Week \& Space Technology, July 27 1995, pp. 56-59.

${ }^{5}$ David, L., "DC-X Completes Pivot Test Over White Sands," Space News, Vol. 6, No. 27, July 10-16 1995 , pp. 1,20 .
${ }^{6}$ Dornheim, M. A., "DC-X Holds Promise; Big Questions Remain," Aviation Week \& Space Technology, August 281995 , pp. 56-59.

${ }^{7}$ O'Leary, C. O., Weir, B., and Walker, J. M., “A New Rig for the Measurement of Rotary and Translational Derivatives," ICAS 94-3.4.1, September 1994. In 19th Congress of the International Council of the Aeronautical Sciences ICAS Proceedings.

${ }^{8}$ Edwards, J. W., and Malone, J. B., "Current Status of Computational Methods for Transonic Unsteady Aerodynamics and Aeroelastic Applications," in AGARD Conference Proceedings: Transonic Unsteady Aerodynamics and Aeroelasticity, no. 507, pp. 1-1-1-24, March 1992.

${ }^{9}$ Batina, J. T., "Implicit Upwind Solution Algorithms for Three-Dimensional Unstructured Meshes," AIA A Journal, Vol. 31, No. 5, May 1993, pp. 801-805. (See also AIAA Paper 92-0447).

${ }^{10}$ Woods, W. C., and Merski, N. R., "Aerodynamic 
Characteristics of a Vertical Takeoff / Vertical Landing (VTVL) Single Stage to Orbit Vehicle from $\mathrm{M}=0.1$ to 10," AIAA Paper 95-1828, 1995.

${ }^{11}$ Steinbrenner, J. P., Chawner, J. R., and Fouts, C. L., "The GRIDGEN 3D Multiple Block Grid Generation System," Wright Research and Development Center Report WRDC-TR-90-3022, October 1989.

${ }^{12}$ Samareh-Abolhassani, J., "GridTool: A Surface Modeling and Grid Generation Tool," in Proceedings of the Workshop on Surface Modeling, Grid Generation, and Related Issues in CFD Solutions, Vol. CP-3291, pp. 821-831, NASA, May 1995.

${ }^{13}$ Peraire, J., Morgan, K., and Peiro, J., "Unstructured Finite Element Mesh Generation and Adaptive Procedures for CFD," in AGARD Conference Proceedings: Application of Mesh Generation to Complex 3-D Configurations, no. 464, pp. 18.1-18.12, 1990.

${ }^{14}$ Kennon, S. R., Meyering, J. M., Berry, C. W., and Oden, J. T., "Geometry-Based Delaunay Tetrahedralization and Mesh Movement Strategies for Multi-Body CFD," AIAA Paper 92-4575, August 1992.

${ }^{15}$ Yoshinaga, T., Tate, A., and Sekine, H., "Side Force of Blunted Circular Cylinders at High Angles of Attack in Supersonic Flow," AIAA Paper 94-3520, August 1994. In AIAA Atmospheric Flight Mechanics Conference Proceedings.

${ }^{16}$ Cobleigh, B. R., "High-Angle-of-Attack Yawing Moment Asymmetry of the X-31 Aircraft from Fligh Test," AIAA Paper 94-1803, June 1994. In 12th AIAA Applied Aerodynamics Conference Proceedings.

${ }^{17}$ Dusing, D. W., and Orkwis, P. D., "On Computing Vortex Asymmetries About Cones at Angle of Attack Using the Conical Navier-Stokes Equations," AIAA Paper 93-3628, August 1993. In AIAA Atmospheric Flight Mechanics Conference Proceedings.

${ }^{18}$ Fisher, D. F., and Cobleigh, B. R., "Controlling Forebody Asymmetries in Flight-Experience with Boundary Layer Transition Strips," AIAA Paper 941826, June 1994. In 12th AIAA Applied Aerodynamics Conference Proceedings.

${ }^{19}$ Newsome, R. W., and Kandil, O. A., "Vortical Flow Aerodynamics-Physical Aspects and Numerical Simulation," AIAA Paper 87-0205, January 1987.

${ }^{20}$ Roe, P. L., "Approximate Riemann Solvers, Parameter Vectors, and Difference Schemes," Journal of Computational Physics, Vol. 43, October 1981, pp. 357372 .

${ }^{21}$ Liou, M.-S., and Christopher J. Steffen, J., "A New Flux Splitting Scheme," NASA TM 104404, May 1991.

${ }^{22}$ van Leer, B., "Flux Vector Splitting for the Euler Equations," Lecture Notes in Physics, No. 170, July 1982, pp. 507-512.

${ }^{23}$ Einfeldt, B., Munz, C. D., Roe, P. L., and Sjögreen, B., "On Godunov-Type Methods near Low Densities,"
Journal of Computational Physics, No. 92, February 1991, pp. 273-295.

${ }^{24}$ van Leer, B., "Towards the Ultimate Conservative Scheme. V. A Second-Order Sequel to Godunov's Method," Journal of Computational Physics, Vol. 32, 1979, pp. 101-136.

${ }^{25}$ Frink, N. T., Parikh, P., and Pirzadeh, S., "A Fast Upwind Solver for the Euler Equations on ThreeDimensional Unstructured Meshes," AIAA Paper 910102, January 1991.

${ }^{26}$ Mulder, W. A., and van Leer, B., "Experiments with Implicit Upwind Methods for the Euler Equations," Journal of Computational Physics, Vol. 59, 1985, pp. 232-246. (See also AIAA Paper 83-1930.).

${ }^{27}$ Jameson, A., Schmidt, W., and Turkel, E., "Numerical Solutions of the Euler Equations by Finite Volume Methods Using Runge-Kutta Time-Stepping Schemes," AIAA Paper 81-1259, June 1981. 